\title{
Digital imaging analysis for the study of endotoxin-induced mitochondrial ultrastructure injury
}

\author{
Mandar S. Joshi ${ }^{\text {a }}$, Elliott D. Crouser ${ }^{\mathrm{b}}$, \\ Mark W. Julian ${ }^{\mathrm{b}}$, Brandon L. Schanbacher ${ }^{\mathrm{a}}$ and \\ John Anthony Bauer ${ }^{\mathrm{a}, *}$

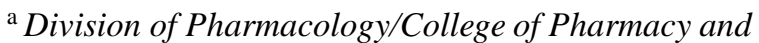 \\ OSU Heart \& Lung Research Institute, The Ohio State \\ University Medical Center, Columbus, OH 43210, \\ USA \\ ${ }^{\mathrm{b}}$ Division of Pulmonary and Critical Care Medicine, \\ Department of Internal Medicine, The Ohio State \\ University Medical Center, Columbus, OH 43210, \\ USA
}

Received 19 October 2000

Accepted 10 November 2000

Primary defects in mitochondrial function have been implicated in over 100 diverse diseases. In situ, mitochondria possess unique and well-defined morphology in normal healthy cells, but diseases linked to defective mitochondrial function are characterized by the presence of morphologically abnormal and swollen mitochondria with distorted cristae. In situ study of mitochondrial morphology is established as an indicator of mitochondrial health but thus far assessments have been via subjective evaluations by trained observers using discontinuous scoring systems. Here we investigated the value of digital imaging analysis to provide for unbiased, reproducible, and convenient evaluations of mitochondrial ultrastructure. Electron photomicrographs of ileal mucosal mitochondria were investigated using a scoring system previously described by us, and also analyzed digitally by using six digital parameters which define size, shape, and electron density characteristics of over 700 individual mitochondria. Statistically significant changes in mitochondrial morphology were detected in LPS treated animals relative to vehicle control using both the subjective scoring system and dig-

${ }^{*}$ Corresponding author: John A. Bauer, Ph.D., Division of Pharmacology/College of Pharmacy, OSU Heart \& Lung Research Institute, The Ohio State University, 412 Riffe Building, 500 West 12th Avenue, Columbus, OH 43210, USA. Tel.: +1 614292 1614; Fax: +1614292 9083; E-mail: Bauer.140@ osu.edu. ital imaging parameters $(p<0.05)$. However, the imaging approach provided convenient and high throughput capabilities and was easily automated to remove investigator influences. These results illustrate significant changes in ileal mucosal mitochondrial ultrastructure during sepsis and demonstrate the value of digital imaging technology for routine assessments in this setting.

Keywords: Digital image analysis, endotoxin, mitochondria, sepsis, ultrastructure

\section{Introduction}

Mitochondria are critical cellular organelles (0.5$1.0 \mu \mathrm{m}$ ) located in the cytoplasm of most eukaryotic cell types. They are responsible for the generation of approximately $90 \%$ of a cell's total ATP supply and therefore serve as the primary source of cellular energy production under aerobic conditions. Traditionally, mitochondria have been viewed as the intracellular "powerhouse". However, recent studies recognize that mitochondria are not merely semiautonomous ATP generators, but highly evolved organelles that are fully integrated into the overall functioning of the eukaryotic cell. $[4,15,19,26]$. It has been recognized since the late 1960 's that mitochondria play a major role in many metabolic processes of eukaryotic cells, including cytosolic calcium homeostasis, modulation of cell redox status, osmotic regulation, $\mathrm{pH}$ control, and intracellular signaling [32]. Mitochondria have also been shown to be involved in apoptotic as well as necrotic cell death $[3,7,10,27]$.

In addition to the realization of essential aspects of mitochondrial performance, the potential roles of mitochondrial dysfunction in disease are increasingly evident $[13,14,17,30]$. Thus far, primary defects in mitochondrial function have been implicated in over 100 diverse diseases, including neurodegenerative disorders [2], liver disease [20], diabetes mellitus [1], bladder dysfunction [18] and cardiac failure [21,22]. We 
have also recently shown the involvement of mitochondria in sepsis-related organ failure [5,6]. Furthermore, the severity of sepsis-induced mitochondrial ultrastructural injury strongly correlates with the impaired oxygen metabolism [16], typical of sepsis, in systemic organs [5]. Thus, mitochondrial dysfunction appears to have a general role in a wide array of pathophysiological settings and may be a new target for therapeutic strategies [17].

Mitochondria have unique and well-defined morphology that includes an outer membrane, which is permeable to most small molecules $(<10 \mathrm{kD})$ and ions, and an extensively invaginated inner membrane (cristae) with more selectivepermeability characteristics [19]. In normal cells, this organelle is typically oval shaped or elongated; however, alterations in permeability, such as during various disease states, cause significant changes in mitochondrial shape $[11,12,20]$. Diseases linked to defective mitochondrial function are characterized by the presence in the tissues of morphologically abnormal and swollen mitochondria with distorted cristae, and the study of in situ mitochondrial morphology can be a suitable indicator of the health and viability of this organelle [24,25,28].

Assessments of in situ mitochondrial morphology have potential value in the study of disease mechanisms and therapeutic interventions, but quantitative and robust methods to do so are not well-defined [15]. In previous reports, we and others have examined in situ mitochondrial morphology via examination of electron photomicrograph images and histopathology scoring approaches [5-7,29]. These qualitative methods, based primarily upon subjective scoring of mitochondrial swelling and morphological changes, have been valuable, but several important limitations exist. These limitations include a required expertise in mitochondrial assessments, potential variability in the results from different laboratories, limited statistical power of discontinuous parameters, and the potential for investigator bias.

Recent advances in digital imaging tools provide an opportunity to characterize images using automated software with high precision, minimum investigator bias, and provision of continuous numerical parameters for statistical comparisons. Digital imaging methods may also increase data throughput and reliability. No previous studies have demonstrated the value of digital imaging technologies for the in situ assessment of mitochondrial morphology, and optimal conditions to do so have not been defined. Here, we investigated the potential value of using digital imaging analysis to provide for unbiased, reproducible, and convenient evaluations of mitochondrial ultrastructure, as captured in electron photomicrographs of ileal mucosal tissue taken from Control and endotoxin (LPS)-treated animals.

\section{Methods}

\subsection{Study design and tissue collection}

Ileal mucosal tissues were harvested from a feline model of lipopolysaccharide (LPS)-induced organ failure $[5,6]$. Segments of ileal tissue were collected at 2 and 4 hours after LPS $(3.0 \mathrm{mg} / \mathrm{ml}$, IV) or saline (vehicle) administration and then processed for electron microscopy (see below). Electron photomicrographs of ileal mitochondria were then studied using a digital imaging approach and a traditional scoring system we previously developed $[5,6]$. The animal preparation used has been described in detail previously [5,6], and all experiments were approved by The Ohio State University Institutional Laboratory Animal Care and Use Committee.

\subsection{Tissue collection and electron microscopy}

Ileal tissue samples were diced and immediately submerged into buffered isotonic fixative (4\% paraformaldehyde, $2.5 \%$ glutaraldehyde, and $0.1 \mathrm{M}$ sucrose in $0.1 \mathrm{M}$ phosphate buffer, $\mathrm{pH}=7.4$ ) for approximately 2 hours at room temperature. The tissue was then minced such that longitudinal sections through the villi were easily obtained. Tissue pieces were then repeatedly rinsed in isotonic buffer $(0.1 \mathrm{M}$ sucrose in $0.1 \mathrm{M}$ phosphate buffer, $\mathrm{pH}=7.4$ ), postfixed in $1 \%$ osmium tetroxide in rinse buffer for 1 hour at room temperature, rinsed again in rinse buffer, and stored overnight at $4^{\circ} \mathrm{C}$. The following morning, the tissue pieces were allowed to come to room temperature, then dehydrated through an ascending series of ethanol solutions. After rinsing with propylene oxide, they were infiltrated with and embedded in Spurr media that polymerized overnight at $60^{\circ} \mathrm{C}$. The next day, thin sections $(80-90 \mathrm{~nm})$ were cut on a Reichart Ultracut E microtome, mounted on copper grids, stained with $2 \%$ uranyl acetate and Reynolds lead citrate, and then later examined using a Phillips CM-12 transmission electron microscope at $60 \mathrm{kV}$. All electron photomicrograph images investigated in this study had a magnification of $55,000 \times[5,6]$. 
Table 1

Qualitative scoring system*

\begin{tabular}{|c|c|c|}
\hline $\begin{array}{l}\text { Cellular injury } \\
\text { stage }\end{array}$ & $\begin{array}{l}\text { Mitochondrial } \\
\text { injury score }\end{array}$ & Mitochondrial characteristics \\
\hline Stage I & 0 & Normal appearance \\
\hline Stage II/III & 1.0 & $\begin{array}{l}\text { Swelling of endoplasmic reticulum, minimal } \\
\text { mitochondrial swelling }\end{array}$ \\
\hline \multirow[t]{3}{*}{ Stage IV } & 2.0 & Mild mitochondrial swelling \\
\hline & 3.0 & Moderate or focal high-amplitude swelling \\
\hline & 4.0 & $\begin{array}{l}\text { Diffuse high-amplitude swelling, disruption of cristal } \\
\text { membrane integrity }\end{array}$ \\
\hline Stage V/VI & 5.0 & $\begin{array}{l}\text { High-amplitude swelling with some mitochondrial } \\
\text { flocculent densities and/or calcifications }\end{array}$ \\
\hline \multicolumn{3}{|c|}{ Digital imaging parameters } \\
\hline $\begin{array}{l}\text { Qualitative } \\
\text { parameter }\end{array}$ & $\begin{array}{c}\text { Characteristic } \\
\text { morphological } \\
\text { change }\end{array}$ & $\begin{array}{l}\text { Digital imaging } \\
\text { parameter }\end{array}$ \\
\hline Swelling & $\begin{array}{l}\text { Increased size } \\
\text { change in shape }\end{array}$ & $\begin{array}{l}\text { - Mitochondrial area } \\
\text { - Aspect ratio } \\
\text { - Roundness }=\text { perimeter }^{2} /(4 \cdot \pi \cdot \text { area }) \\
\text { - Perimeter }\end{array}$ \\
\hline Condensation & $\begin{array}{l}\text { Change in } \\
\text { intracristal structure }\end{array}$ & $\begin{array}{l}\text { - Mean optical density } \\
\text { - Variation in optical density (OD-CV\%) }\end{array}$ \\
\hline
\end{tabular}

$*[5,6,29] ;{ }^{\ddagger}[8,9,29]$.

\subsection{Morphological assessments of mitochondria}

\subsubsection{Qualitative morphological scoring}

High resolution black and white electron photomicrographs $(8.5 \times 11$ inches $)$ were evaluated using a strategy designed to consistently grade the severity of mitochondrial ultrastructural injury, as previously described [5,6]. The association between the degree of mitochondrial injury and the defined stages of cellular injury [29] was employed to quantify the severity of ultrastructural injury to the ileal mitochondria on a scale of $0-5$, as shown in Table 1. This approach is similar to the morphometric analysis developed by Trimmer et al. (2000). Using this scoring approach, we have recently demonstrated evidence of mitochondrial ultrastructural injury at 2 and 4 hours post-LPS in this feline model $[5,6]$.

\subsubsection{Digital imaging evaluations}

Electron photomicrographs $(55,000 \times$ magnification $)$ were converted to computer files using a high-resolution flatbed scanner (Hewlett-Packard ScanJet 6200C, 2400 dpi, TIF file format). The outlines of all mitochondria present in each image (roughly 10 per photomicrograph) were traced by automated edge detection us- ing ImagePro 4.0 software (Media Cybernetics, Silver Springs, MD). Once traced, each individual mitochondrion was independently evaluated using digital parameters. All subsequent image analysis was also performed using ImagePro 4.0 software.

Traditional evaluations of mitochondrial morphology focus mainly upon the size and shape of the mitochondria and the structure of the cristae. We therefore selected six digital parameters that related to these subjective scoring parameters (Table 1, see Digital Imaging Parameters). Measurements of these six parameters were then made on each individual mitochondrion (e.g., approximately 700 mitochondria were individually investigated). Average parameter values were then determined for each electron photomicrograph image. To enhance automation, these six parameters were defined in the imaging software, and a macro-subroutine was programmed to allow high-throughput parameter measurements for all collected images.

\subsection{Statistical analyses}

All data are expressed as the mean \pm SEM. All statistical tests were conducted using SigmaStat software. Comparisons of parameters between groups were con- 


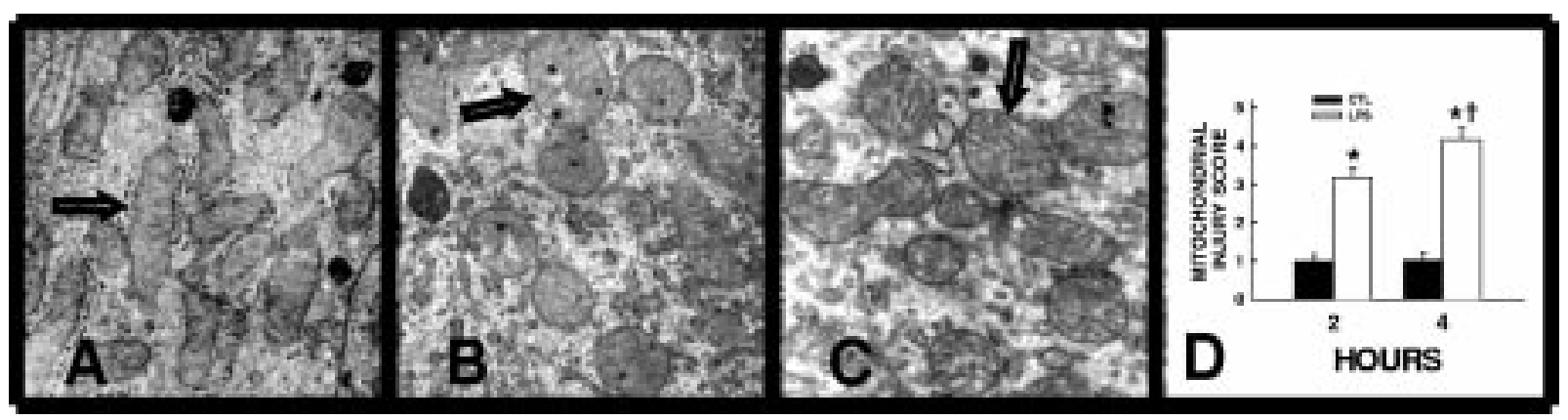

Fig. 1. Representative electron photomicrographs of ileal mucosal mitochondria. (A) Control animal 4 hours post-treatment; (B) LPS-treated animal 2 hours post-treatment; (C) LPS-treated animal 4 hours post-treatment; (D) Injury scores as determined using qualitative criteria defined in Table 1 (mean \pm SEM). In comparison to the normal mitochondrial ultrastructure in control, LPS treatment caused significant ultrastructural injury (see arrows in (B), (C)). Mild to moderate mitochondrial swelling was typical of most of the LPS-treated animals by 2 hours (B), while high amplitude swelling with concomitant loss of membrane integrity was often observed by 4 hours post-treatment (C). (Magnification: 55,000×; Staining: $2 \%$ uranyl acetate and Reynolds lead citrate). Significant mitochondrial ultrastructural injury was apparent within 2 hours and worsened by 4 hours following LPS treatment $\left({ }^{*} p<0.001\right.$, compared to time-matched Controls; ${ }^{\dagger} p<0.01$, compared to corresponding 2-hour measurement).

ducted using 2-factor ANOVA (testing for time and treatment effects), followed by Newman-Keuls posthoc tests. Pearson's nonparametric correlation analyses were used to test for statistically significant associations between qualitative mitochondrial injury scores and quantitative morphological parameters. In all cases, $p<0.05$ was considered statistically significant.

\section{Results}

As shown previously [5,6], significant mitochondrial ultrastructural injury was evident in ileal mucosal tissues 2 hours following LPS treatment (Fig. 1). Mitochondrial injury typically consisted of mild to moderate swelling of all intra-mitochondrial compartments by 2 hours with high amplitude swelling and accompanying loss of membrane integrity evident by 4 hours post-treatment (Fig. 1). Qualitative assessment of mitochondrial injury (using criteria defined in Table 1) following LPS administration, yielded a mean injury score that was 4 times greater than that of timematched Controls by 4 hours (Fig. 1, lower panel).

Shown in Fig. 2 are representative electron photomicrograph images of single ileal mitochondria 4 hours after Control or LPS treatment. In addition, mean values of the six digital parameters (as listed in Table 1) derived in this study are provided. In preliminary studies, we investigated the variation of the mitochondrial imaging parameters. Three separate investigators (blinded to the treatment groups) studied a subset of electron photomicrographs on three separate occasions to derive inter- and intra-investigator parameter variabilities. In all cases and for all 6 digital parameters, the coefficient of variation was less than $4 \%$.

In our preliminary investigations, we found our digital imaging approach to be convenient and time efficient. Following the establishment of optimal conditions, images could be analyzed at the rate of about 1 image $/ 5$ min. Due to the automation, all the data was directly obtained in a form easy for statistical analysis (spreadsheet format). In total, about 700 individual mitochondria were analyzed in the present study.

The mean mitochondrial morphological assessments for the Control and LPS-treated groups over time are shown in Fig. 3. We observed statistically significant differences between the groups with respect to $\mathrm{Mi}$ tochondrial Area, Aspect Ratio, Perimeter, and mean Optical Density (OD) at both the 2 and 4 hour timepoints. Roundness and the Variation in Optical Density (OD-CV\%) were significantly different between the groups as well, but only at the 2 hour time-point. These statistically significant differences are consistent with previous findings wherein LPS treatment caused time-dependent mitochondrial ultrastructural injury in ileal tissues [6].

Shown in Fig. 4 are the relationships between the six digital mitochondrial parameters and the qualitative assessments of mitochondrial injury in the same electron photomicrograph images according to Table 1. Using Pearson's nonparametric correlation analysis, statistically significant correlations were observed for mitochondrial Area, Aspect Ratio, Roundness, and Perimeter; whereas, the OD parameters did not demonstrate significant correlations. 


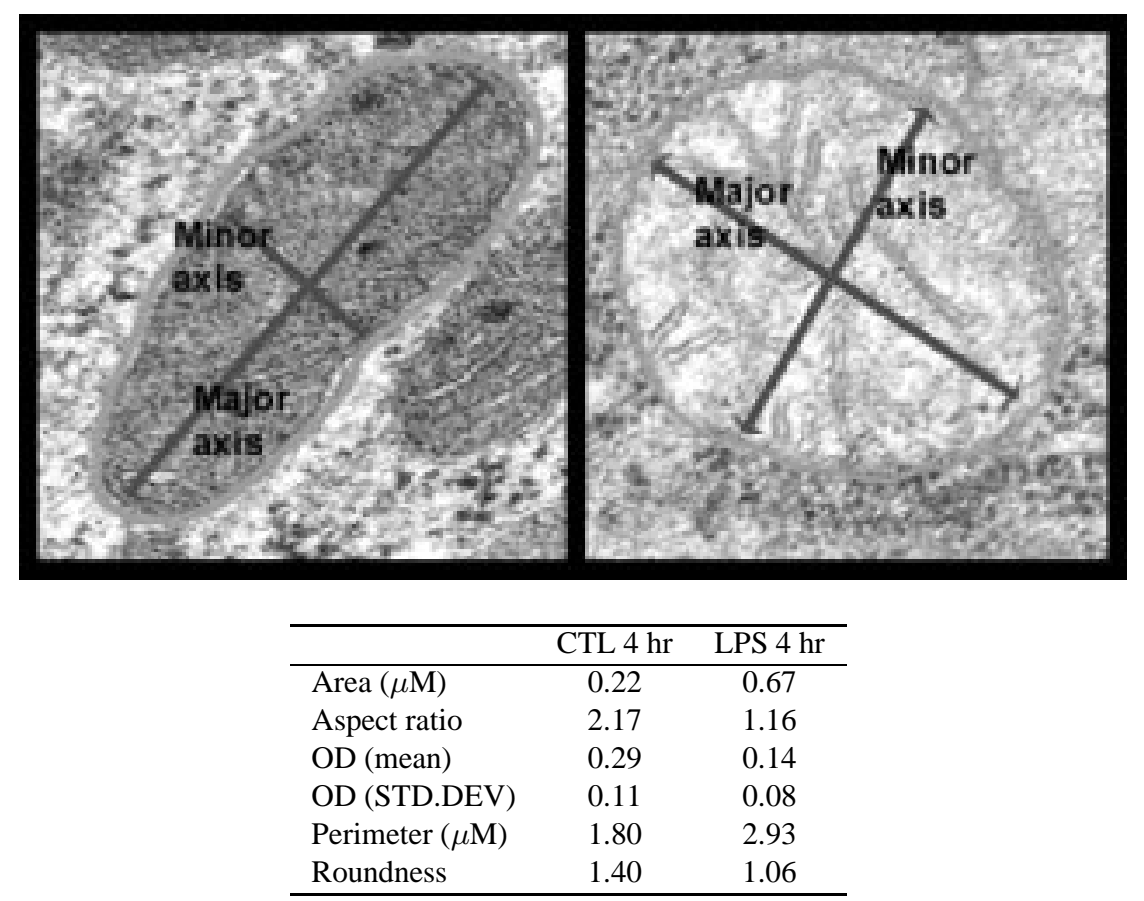

Fig. 2. Representative of digital imaging parameters for individual mitochondria 4 hours after the administration of vehicle control (left panel) or LPS (right panel). Digital parameters of area and perimeter were derived from a continuous tracing of the outer mitochondrial membrane (green line). The aspect ratio and roundness of individual mitochondria were determined using the major and minor axes (red lines), as shown. The optic density (OD) parameters were measured within the region described by the perimeter (green line) and likely reflect changes in the density of the mitochondrial matrix (e.g., a decrease in OD may occur during osmotic swelling).
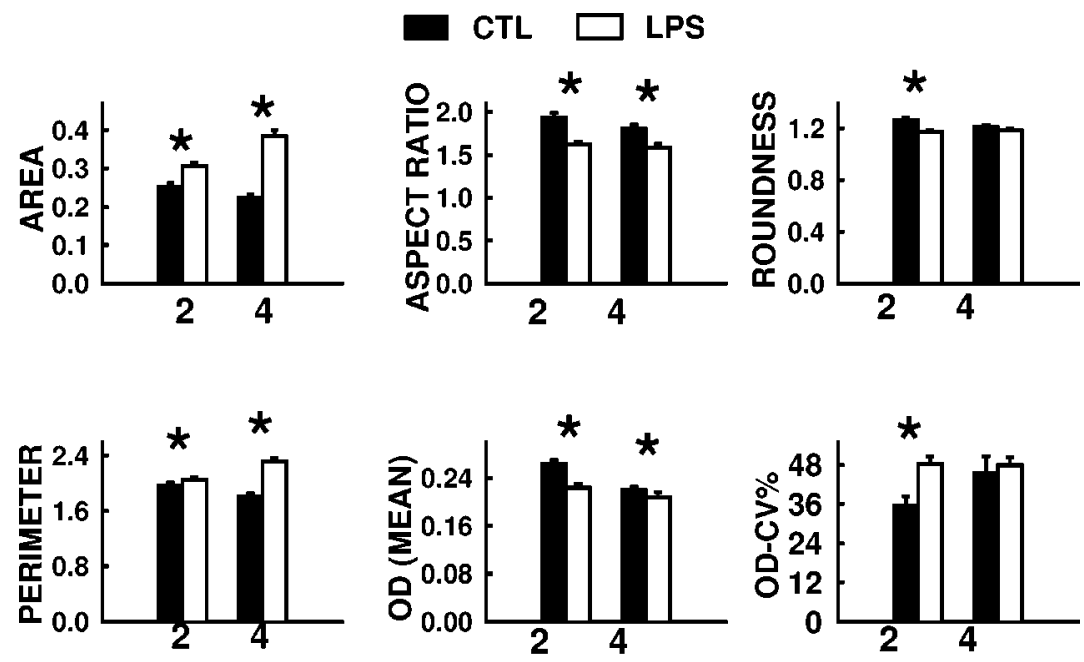

\section{HOURS}

Fig. 3. Mean morphological assessments 2 and 4 hours after the administration of vehicle control or LPS. Two hours after LPS treatment, all measured morphological parameters demonstrated significant deviations from the Controls. LPS-induced changes in mitochondrial morphology included an increase in size, as reflected by greater perimeter and area measurements, and a more rounded shape, as reflected by a decrease in the aspect and roundness measurements. The mean optic density (OD) was reduced with the variation in optic density (OD-CV\%) increased 2 hours post-treatment, perhaps related to the osmotic swelling of the mitochondria. Morphological parameters of area, aspect ratio, perimeter, and OD remained significantly different between the groups at 4 hours post-treatment. 

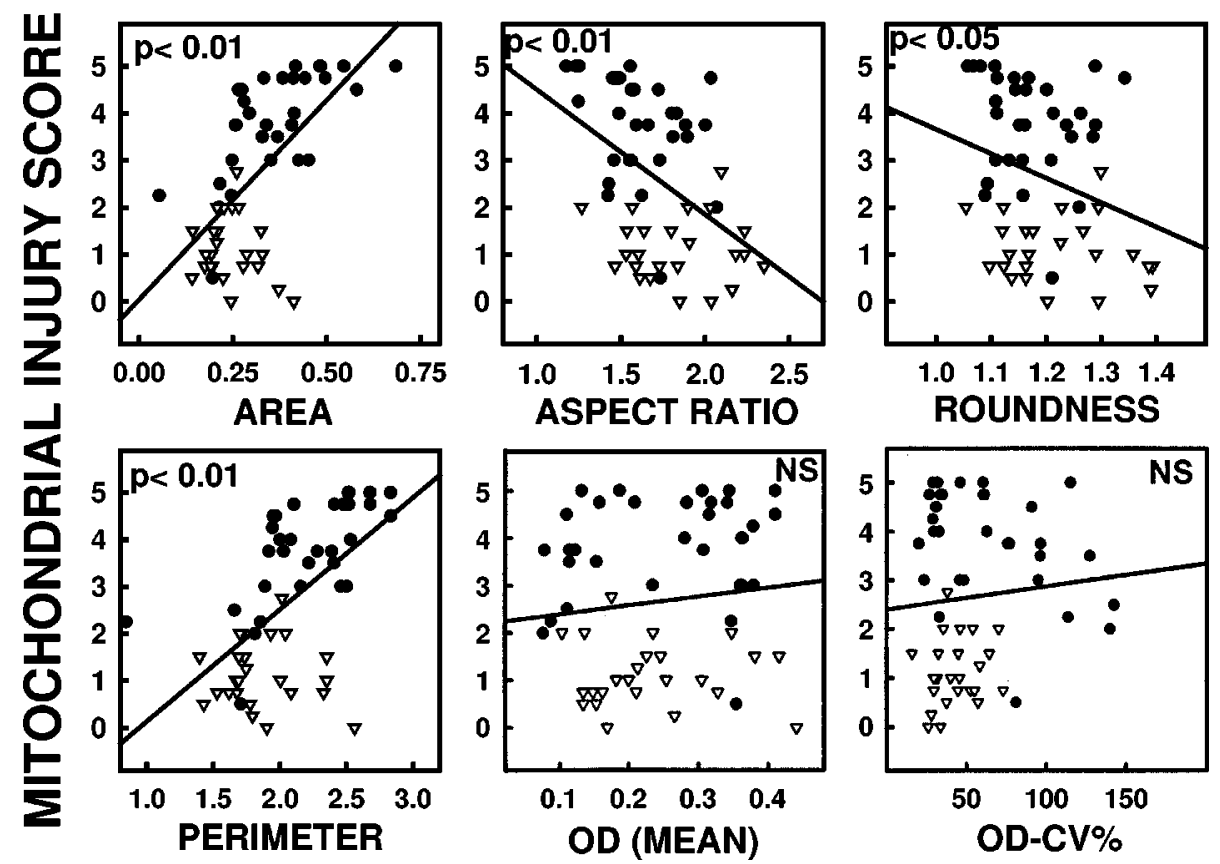

Fig. 4. Relationship between the mitochondrial injury score and the digital imaging parameters assessed 4 hours after the treatment with LPS (black circles) and in time-matched Controls (open triangles). Evaluations of mitochondrial ultrastructure using the qualitative scoring method (i.e., mitochondrial injury scores) were highly correlated with respect to changes in area, aspect ratio, roundness and perimeter, but not with the OD parameters.

\section{Discussion}

Mitochondria are now recognized as important participants in a variety of acute and chronic disease states. In this regard, in situ mitochondrial morphology closely correlates with changes in oxygen metabolism in systemic organs [5] and accurately reflects the severity of cell damage [29]. The use of electron microscopy for the purpose of mitochondrial visualization has been conducted for several decades but only recently have investigators attempted to use these images for morphological assessments in pathological settings. Thus far, all previous reports have used qualitative scoring approaches to assess in situ mitochondrial health. While valuable, these approaches require investigator expertise, are labor intensive, provide discontinuous parameters, and are difficult to standardize. We therefore investigated the use of digital imaging technologies to aid in throughput and reliability for mitochondrial morphology studies. We identified six quantitative parameters that were consistent with the ultrastructural hallmarks of mitochondrial damage, includingswelling and condensation. These parameters were easily assessed using digital imaging software and throughput was greatly facilitated by the creation of an automated subroutine. Further advantages included a high degree of reproducibility (inter- and intra-observer variation $<4 \%$ ) and the generation of continuous parameters for statistical analyses.

Using a qualitative scoring system, we have recently reported time-dependent changes in ileal mitochondrial ultrastructure following LPS treatmentin cats [5, $6]$. In the present study, we found that four of the six digital parameters we investigated provided statistical conclusions consistent with our previous report. These objective parameters are reflective of changes in size and shape (i.e., mitochondrial area, aspect ratio, perimeter, and roundness). These size- and shaperelated mitochondrial parameters had a high degree of correlation to the scoring system we previously employed. In contrast, parameters that reflected intramitochondrial density and condensation were different between the groups only at 2 hours post-treatment, and these parameters did not correlate with the mitochondrial injury score. This discrepancy suggests that characteristics of condensation may be less sensitive than changes in size and/or shape in this model of mitochondrial injury. Alternatively, the subjective assess- 
ments utilized for determining the mitochondrial injury scores may have been derived more from shape changes than from density changes.

In summary, we have developed and validated a digital imaging approach for routine morphological assessments of mitochondria from electron photomicrograph images. Several scalar parameters were readily derived to provide data regarding mitochondrial size, shape, and ultrastructural density. These parameters provided statistical conclusions identical to an integrated mitochondrial injury scoring system derived from more labor-intensive subjective assessments. Most digital parameters were highly correlated to the subjective scoring system. This concurrence provides evidence of the robust value of the digital methods and further validates the conclusions previously derived using the subjective scoring system. Thus, the primary advantages of the digital imaging approach are enhanced throughput, a high degree of standardization, and an excellent concurrence to other established methodologies. These results emphasize the utility of mitochondrial ultrastructural evaluations in the setting of disease and demonstrate the value of digital imaging methods for this purpose.

\section{Acknowledgements}

This work was supported in part by grants from the National Institutes of Health (HL59791, HL63067, and DK55053 awarded to JAB; HL04335 awarded to EDC) and the American Heart Association (0051013B awarded to EDC).

\section{References}

[1] C.M. Anderson, Mitochondrial dysfunction in diabetes mellitus, Drug Development Research 46 (1999), 67-79.

[2] M. Arai, H. Imai, T. Koumura, M. Yoshida, K. Emoto, M. Umeda, N. Chiba and Y. Nakagawa, Mitochondrial phospholipid hydroperoxide glutathione peroxidase plays a major role in preventing oxidative injury to cells, J. Biol. Chem. 274 (1999), 4924-4933.

[3] K.M. Broekemeier, C.K. Klocek and D.R. Pfeiffer, Proton selective substate of the mitochondrial permeability transition pore: regulation by the redox state of the electron transport chain, Biochemistry 37 (1998), 13 059-13065.

[4] J.M. Collombet and C. Coutelle, Towards gene therapy of mitochondrial disorders, Mol. Med. Today 4 (1998), 31-38.

[5] E.D. Crouser, M.W. Julian and P.M. Dorinsky, Ileal VO(2)$\mathrm{O}(2)$ alterations induced by endotoxin correlate with severity of mitochondrial injury, Am. J. Respir. Crit. Care Med. 160 (1999), 1347-1353.
[6] E.D. Crouser, M.W. Julian, D.M. Weinstein, R.J. Fahy and J.A. Bauer, Endotoxin-induced ileal mucosal injury and nitric oxide dysregulation are temporally dissociated, Am. J. Respir. Crit. Care Med. 161 (2000), 1705-1712.

[7] E.J. Griffiths, Mitochondria - potential role in cell life and death [In Process Citation], Cardiovasc. Res. 46 (2000), 24-27.

[8] C.R. Hackenbrock, Ultrastructural bases for metabolically linked mechanical activity in mitochondria. I. Reversible ultrastructural changes with change in metabolic steady state in isolated liver mitochondria, J. Cell. Biol. 30 (1966), 269-297.

[9] C.R. Hackenbrock, Ultrastructural bases for metabolically linked mechanical activity in mitochondria. II. Electron transport-linked ultrastructural transformations in mitochondria, J. Cell. Biol. 37 (1968), 345-369.

[10] A.P. Halestrap, P.M. Kerr, S. Javadov and S. Suleiman, The mitochondrial permeability transition: role in ischemia/reperfusion injury, Sepsis 2 (1998), 313-325.

[11] L. Jia, R.R. Dourmashkin, A.C. Newland and S.M. Kelsey, Mitochondrial ultracondensation, but not swelling, is involved in TNF alpha-induced apoptosis in human T-lymphoblastic leukaemic cells, Leuk. Res. 21 (1997), 973-983.

[12] L.V. Johnson, M.L. Walsh and L.B. Chen, Localization of mitochondria in living cells with rhodamine 123, Proc. Natl. Acad. Sci. USA 77 (1980), 990-994.

[13] N.G. Larsson and R. Luft, Revolution in mitochondrial medicine, FEBS Lett. 455 (1999), 199-202.

[14] R. Luft, The development of mitochondrial medicine, Proc. Natl. Acad. Sci. USA 91 (1994), 8731-8738.

[15] Mannella, Introduction: Our changing views of mitochondria, J. Bioenerg. Biomembr. 32 (2000), 1-4.

[16] S. Melov, P. Coskun, M. Patel, R. Tuinstra, B. Cottrell, A.S. Jun, T.H. Zastawny, M. Dizdaroglu, S.I. Goodman, T.T. Huang, H. Miziorko, C.J. Epstein and D.C. Wallace, Mitochondrial disease in superoxide dismutase 2 mutant mice, Proc. Natl. Acad. Sci. USA 96 (1999), 846-851.

[17] M.P. Murphy, Selective targeting of bioactive compounds to mitochondria, Trends Biotechnol. 15 (1997) 326-330.

[18] C.A. Nevel-McGarvey, R.M. Levin, N. Haugaard, X. Wu and A.P. Hudson, Mitochondrial involvement in bladder function and dysfunction, Mol. Cell. Biochem. 194 (1999), 1-15.

[19] P.L. Pedersen, Mitochondrial events in the life and death of animal cells: a brief overview, J. Bioenerg. Biomembr. 31 (1999), 291-304.

[20] D. Pessayre, A. Mansouri, D. Haouzi and B. Fromenty, Hepatotoxicity due to mitochondrial dysfunction, Cell Biol. Toxicol. 15 (1999), 367-373.

[21] H.N. Sabbah, V. Sharov, J.M. Riddle, T. Kono, M. Lesch and S. Goldstein, Mitochondrial abnormalities in myocardium of dogs with chronic heart failure, J. Mol. Cell. Cardiol. 24 (1992), 1333-1347.

[22] K. Schulze, A. Dorner and H.-P. Schulthei, Mitochondrial function in heart failure, Heart Failure Reviews 4 (1999), 229-244.

[23] V.P. Skulachev, Mitochondrial physiology and pathology; concepts of programmed death of organelles, cells and organisms, Mol. Aspects Med. 20 (1999), 139-184.

[24] W.C. Stanley and C.L. Hoppel, Mitochondrial dysfunction in heart failure: potential for therapeutic interventions? [editorial; comment], Cardiovasc. Res. 45 (2000), 805-806. 
[25] Y. Sudarikova, L.E. Bakeeva and V.G. Tsiplenkova, Ultrastructure of mitochondrial reticulum of human cardiomyocytes in alcohol cardiomyopathy, Biochemistry (Mosc.) 62 (1997), 9891002 .

[26] S. Sweet and G. Singh, Changes in mitochondrial mass, membrane potential, and cellular adenosine triphosphate content during the cell cycle of human leukemic (HL-60) cells, J. Cell. Physiol. 180 (1999), 91-96.

[27] K. Thress, S. Kornbluth and J.J. Smith, Mitochondria at the crossroad of apoptotic cell death, J. Bioenerg. Biomembr. 31 (1999), 321-326.

[28] P.A. Trimmer, R.H. Swerdlow, J.K. Parks, P. Keeney, J.P.J. Bennett, S.W. Miller, R.E. Davis and W.D.J. Parker, Abnormal mitochondrial morphology in sporadic Parkinson's and
Alzheimer's disease cybrid cell lines, Exp. Neurol. 162 (2000), 37-50.

[29] B.F. Trump, I.K. Berezesky, K.U. Laiho, A.R. Osornio, W.J. Mergner and M.W. Smith, The role of calcium in cell injury. A review, Scan. Electron. Microsc. 62 (1980), 437-492.

[30] D.C. Wallace, Mitochondrial diseases in man and mouse, Science 283 (1999), 1482-1488.

[31] D.C. Wallace, M.D. Brown and M.T. Lott, Mitochondrial DNA variation in human evolution and disease, Gene 238 (1999), 211-230.

[32] K.B. Wallace, J.T. Eells, V.M. Madeira, G. Cortopassi and D.P. Jones, Mitochondria-mediated cell injury. Symposium overview, Fundam. Appl. Toxicol. 38 (1997), 23-37. 


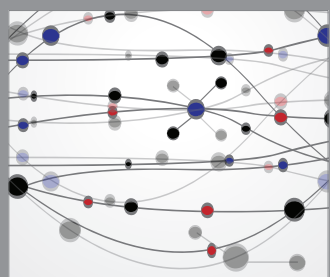

The Scientific World Journal
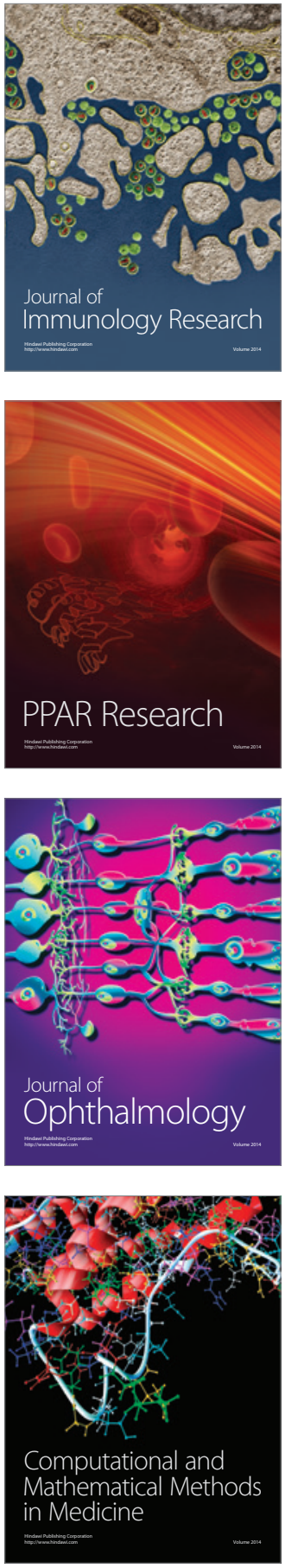

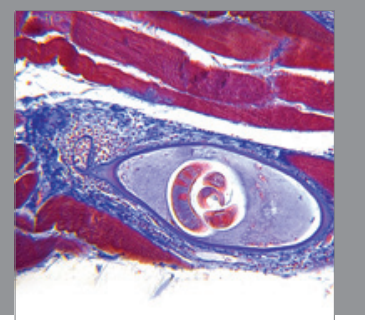

Gastroenterology

Research and Practice
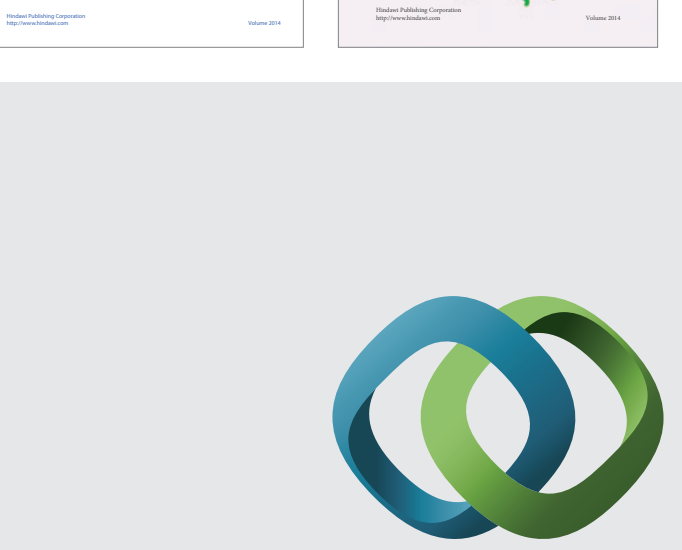

\section{Hindawi}

Submit your manuscripts at

http://www.hindawi.com
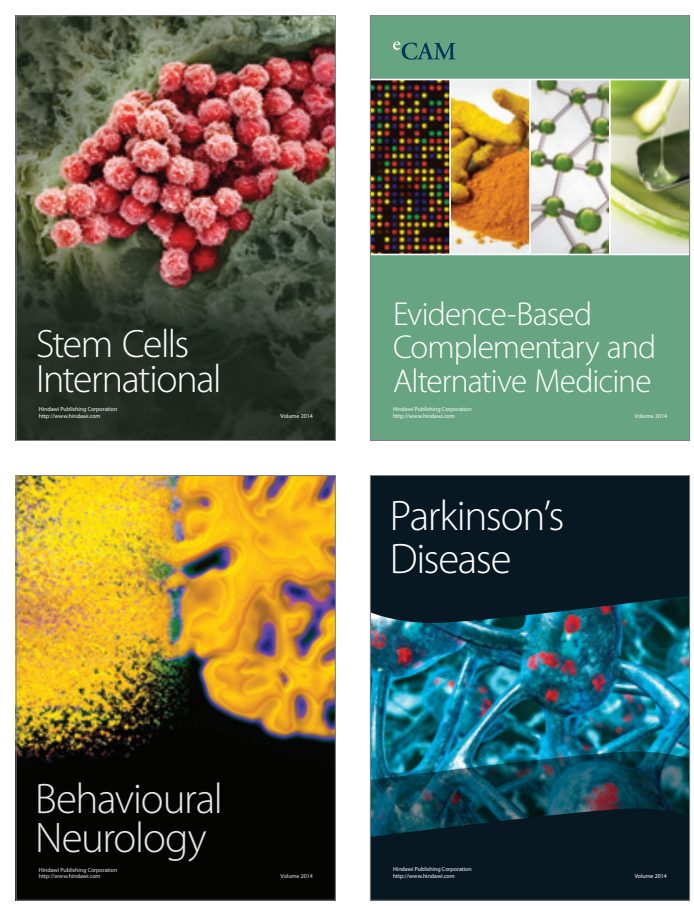

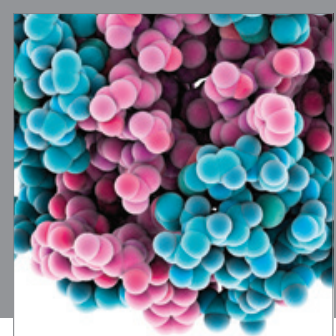

Journal of
Diabetes Research

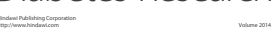

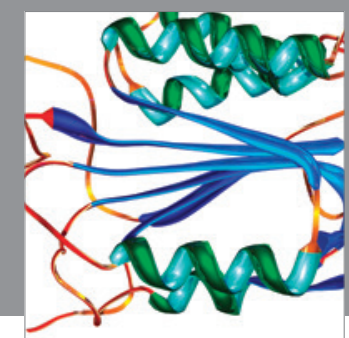

Disease Markers
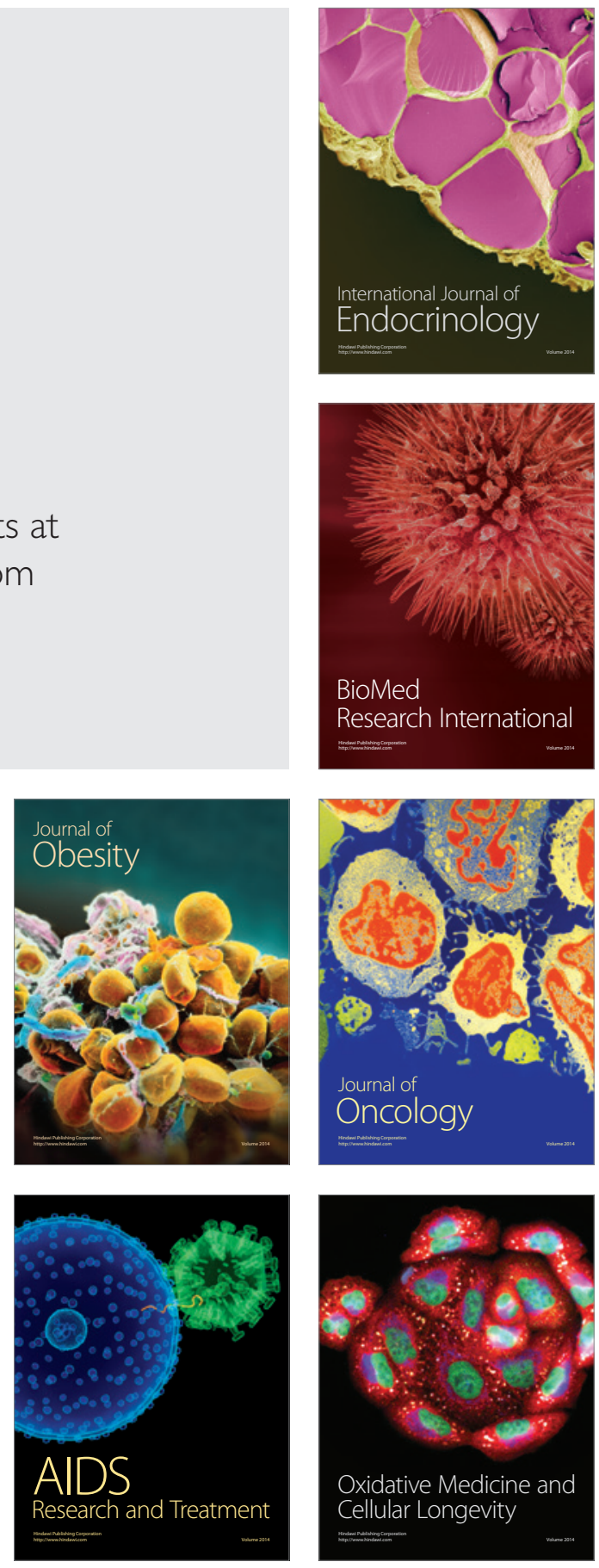